\title{
Chronic meningoencephalitis caused by Echo virus 6 in a patient with common variable immunodeficiency
}

\section{Successful treatment with pleconaril}

\author{
Igor Radanović · Deni Rkman · Paulo Zekan · Marko Kutleša · Bruno Baršić
}

Received: 18 July 2017 / Accepted: 13 October 2017 / Published online: 7 November 2017

(c) Springer-Verlag GmbH Austria 2017

\begin{abstract}
Summary Chronic enteroviral meningoencephalitis, most commonly caused by echoviruses, can particularly be seen in agammaglobulinemic patients. In spite of the fact that no specific treatment for enteroviral infections exists, pleconaril is an antiviral drug reported to be efficient against enteroviral infections in infants and adults. We present a case of a 42-year-old male, previously diagnosed with common variable immunodeficiency, who presented with severe chronic meningoencephalitis caused by Echo virus 6 and was successfully treated with pleconaril. Enteroviruses usually cause mild symptoms, but some strains can cause life-threatening conditions especially in immunocompromised patients. Although pleconaril production is unprofitable due to the rarity of severe disease, our effective treatment should encourage further availability of pleconaril.
\end{abstract}

\author{
I. Radanović · D. Rkman · P. Zekan \\ School of Medicine, University of Zagreb, Šalata 3b, Zagreb, \\ Croatia \\ I. Radanović \\ igor.radanovic@student.mef.hr \\ D. Rkman \\ deni.rkman@student.mef.hr \\ P. Zekan \\ paulo.zekan@student.mef.hr \\ M. Kutleša $(\bowtie) \cdot$ B. Baršić \\ School of Medicine, University Hospital for Infectious \\ Diseases “Dr. Fran Mihaljević", Department of Intensive \\ Care Medicine and Neuroinfectology, University of Zagreb, \\ Mirogojska cesta 8, Zagreb, Croatia \\ marko.kutlescha@gmail.com \\ B. Baršić \\ bbarsic@bfm.hr
}

Keywords Enterovirus - Echoviruses - Meningoencephalitis · Pleconaril · CVID

\section{Introduction}

Immunodeficient patients are known to have a high susceptibility for the development of severe enteroviral (EV) infections of the central nervous system (CNS). Chronic EV meningoencephalitis can particularly be seen in agammaglobulinemic patients. It is most commonly caused by echoviruses, although some other cases have also been reported [1-4]. Common variable immunodeficiency (CVID) is a type of primary immunodeficiency characterized by a large heterogeneity in clinical presentation. It is diagnosed by reduced serum levels of IgG, IgA levels with more than two standard deviations from the normal values and without the presence of any other known cause of hypogammaglobulinemia (e.g. X-linked agammaglobulinemia) [5, 6]. Apart from chronic meningoencephalitis, other EV diseases in patients with CVID have also been reported, such as a recurrent case of hand, foot and mouth disease [7]. In spite of possible fatal outcome, there is no specific treatment for EV infections; however, pleconaril is a drug with reported antienteroviral effects. Pleconaril binds to a hydrophobic pocket in the viral capsid inducing conformational changes, which lead to altered receptor binding and virus uncoating. Furthermore, it achieves several fold higher concentrations within the CNS than in serum, which is highly desirable for the drug targeted toward viruses known to cause CNS infections [8]. Concomitant treatment with pleconaril and intravenous immunoglobulin (IVIG) is reported to be successful in solid organs of adult recipients [9]. There is also a report of in vitro but not in vivo efficacy of IVIG and pleconaril therapy in infants [10]. Monotherapy with pleconaril is reported 
Table 1 Laboratory results obtained during treatment

\begin{tabular}{|l|l|l|l|}
\hline Method/date & Cerebrospinal fluid & Serum & \multicolumn{1}{l}{ Stool } \\
\hline PCR-2/9/2016 & Positive & Positive & - \\
\hline Viral culture-2/9/2016 & Echo virus 6 & Not done & Echo virus 6 \\
\hline PCR-2/19/2016 & Positive for enteroviruses & Positive for enteroviruses & - \\
\hline Viral culture-2/19/2016 & Not done & Not done & Not done \\
\hline 14 day pleconaril started on 2/9/2016 & & & \\
\hline PCR—-3/7/2016 & Negative & Negative & - \\
\hline Viral culture-3/7/2016 & Negative & Not done & Negative \\
\hline PCR-6/9/2016 & Negative & Negative & - \\
\hline Viral culture-6/9/2016 & Negative & Not done & Negative \\
\hline
\end{tabular}

to be beneficial in infants with severe EV infections $[3,11]$. In order to provide evidence for the efficiency of pleconaril treatment in EV meningitis and sepsis in infants, two double-blinded randomized studies have been conducted. The self-limiting and benign nature of EV meningitis was a limiting factor in one study and the other one proved significant virologic but not clinical efficiency of pleconaril treatment [12, 13]. Reports of pleconaril therapy for EV infections in adults are scarce; however, several variably successful pleconaril treatments in patients with EV meningoencephalitis and hypogammaglobulinemia have been reported $[14,15]$.

We present a case of a 42 -year-old male with previously diagnosed CVID who presented with severe chronic meningoencephalitis caused by Echo virus 6 and was successfully treated with pleconaril.

\section{Patient and methods}

A 42-year-old patient was admitted due to chronic meningoencephalitis. The patient had been diagnosed with CVID since 1994 and treated with IVIG monthly ever since. The current illness started 5 weeks prior to admission with fever, headache, gait instability and intermittent dysphasia. At admission the vital signs were normal with jaundice and general malaise. Work-up revealed low white blood cell and low platelet counts of 2.7 and 71 , respectively. The bilirubin was 120 (70 conjugated), aspartat aminostransferase $531 \mathrm{IU} / \mathrm{L}$, alanin aminotransferase $662 \mathrm{IU} / \mathrm{L}$, cerebrospinal fluid (CSF) analysis yielded an increased cell count of $105 / \mathrm{mm}^{3}$ with $82 \%$ mononuclear cells and increased protein content of $1.8 \mathrm{~g} / \mathrm{l}$. Etiology quest revealed that PCR was positive for enteroviral RNA and viral culture detected Echo virus 6. Furthermore, enteroviral RNA could be detected by PCR in blood but the PCR target sequence was a 5'-noncoding region common to all enteroviruses. The patient's IVIG treatment was intensified with a weekly administration of the usual dose ( $0.4 \mathrm{~g}$ per $\mathrm{kg}$ body weight for 5 consecutive days) without any effect and with persistent detection of enteroviral RNA in serum and CSF. Approximately 1 month after admission, we were able to attain ple- conaril (courtesy of Prof. Jacob Westman from Solna, Sweden) and the patient was started on oral treatment ( $3 \times 400 \mathrm{mg}$ daily). The drug provided was in powder form and it had to be prepared before administration.

\section{Results}

After 14 days of pleconaril treatment, enteroviral RNA was not detected in CSF or serum by PCR (Table 1).

Hospitalization of the patient was complicated by the development of severe nosocomial pneumonia and influenza B infections. Due to this unfortunate turn of events, he required mechanical ventilation and tracheostomy. Fortunately, the pneumonia resolved with treatment and we were able to wean him from the ventilator. After 5 months, just prior to discharge from hospital, CSF and serum were analyzed for the presence of enteroviruses and the results of PCR and cultures remained negative and the bilirubin and liver enzymes normalized. At discharge the patient had a Karnofsky performance scale index score of $70 \%$ and was able to walk with assistance.

\section{Discussion}

Enteroviral infections are usually known as mild diseases; however, certain enteroviral strains are more pathogenic and capable of causing severe illness in previously healthy children and adults as well as inducing severe illness in susceptible individuals, especially those with impaired humoral immunity [16]. Despite the fact that some enteroviral strains are resistant to pleconaril, it is most unfortunate that this potentially effective treatment option is not widely available for patients with the severe forms of any enteroviral disease.

Enterovirus 71 (EV 71) could be a life-threatening infection in children. In several well-known epidemics, many of the affected children succumbed to the disease [16, 17]. One of known epidemics was an outbreak of enteroviral infections in children in Kansas City, Missouri in 2014. It was caused by enterovirus D68 and the majority of patients presented with respiratory distress and severe bronchospasms. Pleconaril was not used as a therapy option in those 
epidemics due to suspected resistance [18]; however, due to the effectiveness in vitro, it seems prudent to try pleconaril in all patients with severe enteroviral infections.

Chronic enteroviral meningoencephalitis is a known entity in patients with CVID or Bruton's disease. Untreated disease is usually progressive and often leads to a fatal outcome [19]. According to the literature and our own experience, this subgroup of patients might benefit from pleconaril treatment and should not be deprived of the treatment trial as it can save their lives. Our patient responded well to pleconaril treatment and was able to clear the Echo virus 6 from the blood and CSF. Furthermore, clinical progression of the disease was terminated and his condition improved substantially. Nowadays, the rarity of severe enteroviral disease has rendered the commercial production of pleconaril unprofitable and the drug is therefore unavailable, despite the fact that it has the potential to save lives in specific subgroup of patients; however, due to successful treatment in our patient, we believe that the medical community should endorse and assert the production and availability of pleconaril despite the unlucrative nature of such action.

Acknowledgements We are tremendously thankful to Prof. Jacob Westman for providing pleconaril that cured our patient.

Conflict of interest I. Radanović, D. Rkman, P. Zekan, M. Kutleša, and B. Baršić declare that they have no competing interests.

\section{References}

1. McKinney RE, Katz SL, Wilfert CM. Chronic enteroviral meningoencephalitis in agammaglobulinemic patients. RevInfect Dis. 1987;9(2):334-56.

2. Rudge P, Webster AD, Revesz T, Warner T, Espanol T, Cunningham-Rundles C, et al. Encephalomyelitis in primary hypogammaglobulinaemia. Brain. 1996;119(Pt):1-15.

3. Nowak-Wegrzyn A, Phipatanakul W, Winkelstein JA, Forman MS, Lederman HM. Successful treatment of enterovirus infection with the use of pleconaril in 2 infants with severe combined immunodeficiency. Clin Infect Dis. 2001;32(1):E13-E4.

4. Cunningham CK, Bonville CA, Ochs HD, Seyama K, John PA, Rotbart HA, et al. Enteroviral meningoencephalitis as a complication of X-linked hyper IgM syndrome. J Pediatr. 1999;134(5):584-8.
5. ParkMA,LiJT,HaganJB, MaddoxDE,AbrahamRS.Common variable immunodeficiency: a new look at an old disease. Lancet. 2008;372(9637):489-502.

6. ResnickES, Cunningham-Rundles C. The many faces of the clinical picture of common variable immune deficiency. Curr Opin Allergy Clin Immunol. 2012;12(6):595-601.

7. Le Cleach L, Benchikhi H, Liedman D, Boumsel L, Wolkenstein P, Revuz J. Hand-foot-mouth syndrome recurring during common variable deficiency. Ann Dermatol Venereol. 1999;126(3):251-3.

8. RomeroJR. Pleconaril: a novel antipicornaviraldrug. Expert Opin Investig Drugs. 2001;10(2):369-79.

9. Starlin R, Reed N, Leeman B, Black J, Trulock E, Mundy LM. Acute flaccid paralysis syndrome associated with echovirus 19, managed with pleconaril and intravenous immunoglobulin. Clin Infect Dis. 2001;33(5):730-2.

10. Rentz AC, Libbey JE, Fujinami RS, Whitby FG, Byington CL. Investigation of treatment failure in neonatal echovirus 7 infection. Pediatr Infect Dis J.2006;25(3):259-62.

11. Bauer S, Gottesman G, Sirota L, Litmanovitz I, Ashkenazi S, Levi I. Severe coxsackie virus B infection in preterm newborns treated with pleconaril. Eur J Pediatr. 2002;161(9):491-3.

12. Abzug MJ, Cloud G, Bradley J, Nchez PJS, Romero J, Powell D, et al. Double blind placebo-controlled trial of pleconaril in infants with enterovirus meningitis. Pediatr Infect Dis J. 2003;22(4):335-40.

13. Abzug MJ, Michaels MG, Wald E, Jacobs RF, Romero JR, Sánchez PJ, et al. A randomized, double-blind, placebo-controlled trial of pleconaril for the treatment of neonates with enterovirus sepsis. J Pediatric Infect Dis Soc. 2016;5(1):53-62.

14. Rotbart HA, Webster AD. Pleconaril treatment registry group. Treatment of potentially life-threatening enterovirus infections with pleconaril. Clin Infect Dis. 2001;32:228-35.

15. Wildenbeest JG, Van Den Broek PJ, Benschop KSM, Koen G, Wierenga PC, Vossen ACTM, et al. Pleconaril revisited: clinical course of chronic enteroviral meningoencephalitis after treatment correlates with in vitro susceptibility. Antivir Ther (Lond). 2012;17(3):459-66.

16. Zhang G, Zhou F, Gu B, Ding C, Feng D, Xie F, et al. In vitro and in vivo evaluation of ribavirin and pleconaril antiviral activity against enterovirus 71 infection. Arch Virol. 2012;157(4):669-79.

17. Chia MY, Chiang PS, Chung WY, Luo ST, Lee MS. Epidemiology of enterovirus 71 infections in Taiwan. Pediatr Neonatol. 2014;55(4):243-9.

18. Oermann CM, Schuster JE, Conners GP, Newland JG, Selvarangan R, Jackson MA. Enterovirus D68: a focused review and clinical highlights from the 2014 U.S. outbreak. Ann Am Thorac Soc. 2015;12(5):775-81.

19. Halliday E, Winkelstein J, Webster ADB. Enteroviral infections in primary immunodeficiency (PID): a survey of morbidity and mortality. J Infect. 2003;46(1):1-8. 